\title{
L'ANTHROPOLOGIE ONTOLOGIQUE ET LES VILLES MODERNES: LES APPORTS D'UNE ANTHROPOLOGIE ONTOLOGIQUE AU MILIEU URBAIN.
}

\author{
Thais Cunegatto ${ }^{1}$
}

L'élaboration de cet article a été un grand défi pour moi parce que j'ai eu besoin de me déshabiller de mes outils «classiques» et de revoir l'Anthropologie à partir d'une autre perspective - un exercice toujours nécessaire et productif, mais aussi complexe et parfois douloureux. En mettant en pratique cet exercice de déplacement du chercheur par rapport à ses outils conceptuels et sa pratique, je revisite les auteurs de «l'Anthropologie Contemporaine» pour repenser mes études réaliser dans le milieu urbaine et contemporaine.

Pour commencer ce processus de réflexion, je me demande si la propre classification de ses auteurs ne serait un mouvement contre leurs propres propositions. Est-ce que leurs tentatives de changement épistémologique ne sont pas une rupture contre le système fermé de classification?

Les auteurs contemporains proposent une non dichotomisation du «monde» entre nature et culture. Ses auteurs invitent le chercheur à découvrir le monde de ses interlocuteurs au-delà des conventions, au-delà de la classification préétablie des mondes, au-delà de la recherche d'un universel. Mais comme l'Anthropologie est aussi un domaine qui a été creé appuyé sur la Modernité, elle essaie de toujours tout catégoriser, de mettre les auteurs dans des boîtes fermées, de bien encadrer les sujets et les choses, les chercheurs et leurs domaines de recherche. De cette façon, on commence à classifier les Anthropologies: l'Anthropologie structurale d'un côté, l'Anthropologie contemporaine de l'autre et encore l'Anthropologie post-moderne ou critique. Plusieurs Anthropologies avec leurs plusieurs subdivisions sont toujours bien définies de telle sorte que si le chercheur fait partie d'un domaine particulier de recherche il lui est vraiment interdit de faire partie d'un autre, de même que si une chose appartient au monde de la «nature», elle ne peut pas non plus appartenir au monde de la «culture».

\footnotetext{
${ }^{1}$ l'Université Laval, Quebec, Canadá.
} 
Cette façon de classifier les auteurs est une manière de ne pas s'apercevoir que la «contemporanéité» met en dialogue toutes ces Anthropologies. Ainsi comme Julien (2006) suggère de laisser vide et opérationnel le concept de l'universel, je pense que le concept d'Anthropologie contemporaine est utile jusqu'au moment où on ne le définit pas comme un concept fermé, où il est vide de sens, où l'Anthropologie demeure un devenir.

Ce travail est inspiré dans la proposition d'une rupture du préétabli ; d'une rupture avec ce monde dichotomique et manichéen. Encore en lisant les «contemporains» je pensais que c'était une Anthropologie créée par des chercheurs qui travaillent avec les autochtones, une Anthropologie plutôt ethnologique, qui discutait le «natif loin» : l'Autre qui avait l'altérité bien définie, pouvant être aussi l'Autre qui devient exotique, cela a été la première impression que j'ai eu sur cette approche. En lisant, pourtant plus attentivement et cette fois-ci bien plus sensible, j'ai compris qu'autant qu'anthropologue insérée dans le monde de la modernité, je faisais cette séparation, je mettais les Anthropologies dans des boîtes fermées et je m'interdisais de regarder les mondes différemment. Appuyée sur une citation de l'auteur Viveiros de Castro j'essaie de les sortir de cette boîte et de regarder la complexité des mondes sous les perspectives de cette approche en considérant « la possibilité d'une anthropologie urbaine qui fasse la même chose que l'ethnologie fait, qui étudie les laboratoires de physique, les multinationales pharmaceutiques, les nouvelles technologies de reproduction, les grands courants de pensée dans les universités, la production du discours juridique, politique » (2002: 490). Cette pensée déclenche un replacement du chercheur urbain dans sa façon de faire son métier et son approche avec ses interlocuteurs. C'est à partir de ce replacement que je me lance dans ce travail.

Si nous pensons l'Anthropologie développée par Viveiros de Castro, Tim Ingold, Roy Wagner et Bruno Latour etc., on se rend compte que les questions que ces anthropologues se posent sont toujours pensées à travers la relation du chercheur avec un autre natif lointain, une relation dans laquelle le chercheur et le «natif » partagent des mondes complètement différents. Le but de ce travail est de discuter la manière de penser à partir du «perspectivisme » ou de l'anthropologie symétrique ou même de l'ontologie quand l'autre est notre voisin proche, quand le chercheur et le «natif » cohabitent un «même espace public », quand ils demeurent dans la « même ville », quand la façon de 
regarder, de sentir et de vivre le monde est similaire, mais quand même différente, quand leurs expériences se rapprochent parfois grâce au partage d'une même culture (occidentale et urbaine) et parfois s'éloignent parce que dans cette culture il y a dans beaucoup d'autres cultures.

Les auteurs contemporains balisent une autre pratique anthropologique, une autre façon de regarder l'Autre. Même si en regardant à travers différents points de vue ils veulent saper les dichotomies, les interroger et les démystifier en en retirant le statut de la vérité anthropologique. Les certitudes fondées sur une raison classificatoire du monde ne sont que des projets théoriques qui ont déjà assez pourvu et, par conséquent, elles devraient être remises en question en tant que modes explicatifs de la relation entre l'homme et l'environnement, la culture et la nature, l'esprit et le corps.

Considérés comme écrivains «postmodernes», ces auteurs cherchent à remettre en question la modernité dans leurs productions de connaissances. D'une part, les postulats de ce qu'on appelle les sciences dures et d'autre part, les sciences humaines. C'est-à-dire l'un se situerait du côté de la nature et l'autre de celui de la culture; du sujet et de l'objet. La question qui se pose ici est liée au fait que ces «boîtes» ne sont plus tellement organisées. Alors, quels sont les moyens d'y réussir? Quel est le statut de l'ethnographie? Comment les naturels/ les non-humains s'insèrent-ils dans le monde des humains? Qu'estce l'être humain? Quelles sont les frontières épistémologiques entre les différents domaines du savoir? Quelles sont les limites entre la nature et la culture, l'esprit et le corps, l'individu et la société, moi et l'Autre?

Prenant comme point de départ pour la discussion, je me sers de Todorov, dans le texte intitulé «La découverte de l'Amérique», quand l'auteur écrit à propos de la découverte que le «moi» fait de «l'Autre»: «On peut découvrir les autres en soi, et s'apercevoir que ce n'est pas une substance homogène, et radicalement différente de tout ce qui n'est pas soi-même, moi c'est l'autre. Mais tout autre est un moi aussi » (Todorov 1991: 3.). Todorov choisit de raconter une histoire de la découverte de l'Amérique, parce que c'est une rencontre très étonnante de l'histoire pour parler de cette découverte de l'autre, si chère au travail ethnographique. La rencontre avec l'autre est la condition préalable de travail ethnographique. 
Encore dans le texte Todorov (1991), il y a ce que l'auteur appelle d' « une sorte de parodie du travail ethnographique» qui sert également à réfléchir sur les pièges de l'ethnographie, à partir du moment où Colombo apprend le mot «cacique», il ne se préoccupe plus avec le sens que les autochtones donnent aux mots, mais plutôt à son équivalence en espagnol, en précisant qu'il croit que les autochtones définissent les mêmes distinctions que les Espagnols, sans se rendre compte de la convention, mais dans la naturalité des choses: «Jusque-là, l'amiral n'avait pas été en mesure de comprendre si ce mot (сасіque) signifiait roi ou gouverneur. Ils avaient également un autre mot pour les grands, c'était nitayno mais il ne savait pas si on l'utilisait pour désigner un gentleman, un gouverneur ou un juge » (Todorov, 1991).

C'est dans ce sens que la «traduction» est, comme on a discuté dans les classes du séminaire avancé : Différence et altérité sous l'angle de l'anthropologie ontologique, une tentative d'encadrer l'Autre dans la catégorie de l'homme moderne et occidental. C'est penser que les mondes ailleurs ont toujours une relation avec le monde de l'Occident et partir du principe qu'il y a toujours un universel, que les catégories de pensées sont toujours les mêmes ou plutôt qu'il y la possibilité d'un rapport direct, d'une traduction simple des mots et de la façon d'être au monde des interlocuteurs à travers quelque chose qui soit commune à l'homme occidental. Julien, (2008) en proposant un dialogue intelligent entre les cultures, montre que ce n'est pas une question de traduction, mais de compréhension de l'Autre, de leurs «êtres au monde». Ce dialogue intelligent prend au sérieux le «natif» en comprenant ses catégories de pensée, comme suggère Viveiros de Castro (1998, 2002 et 2009).

Selon Strathern (2006) et Viveiros de Castro (2002) on doit être conscient des «problèmes des natifs», c'est-à-dire plus que discuter la transposition des concepts entre les sociétés - surtout quand il y a la distinction que nous appelons complexe et celles, traditionnelles - nous devons être perspicaces pour apprendre les problèmes qui touchent les interlocuteurs, faire des efforts afin de comprendre une logique différente de celle du chercheur. L'enjeu ici c'est de savoir quand les concepts ne s'appliquent pas dans le terrain de recherche, donc l'accent de Viveiros et Strathern réside sur accorder de l'importance au travail qui est développé sur le terrain en faisant de l'élaboration théorique une étape postérieure qui doit faire référence, qui doit être en «dialogue intelligent» avec toute 
l'expérience ethnographique et non pas l'encadrer, en s'opposant ainsi à l'idée d'aller sur le terrain avec une théorie déjà définie.

La contribution de ces deux auteurs nous transmet encore en héritage l'importance de l'ethnographie pour répondre à ces questions concernant l'expérience ethnographique, elle n'est en contact qu'avec les interlocuteurs qui pourraient avoir une avance théorique. L'activité du travail sur le terrain et la production de la théorie sont deux étapes qui se complètent mutuellement. Dans ce sens, le propos de l'Anthropologie Contemporaine est une plongée dans le monde de l'interlocuteur, elle est un appel à l'ethnographie, au «dialogue intelligent». De cette manière, ce n'est pas une Anthropologie seulement pour l'Autre lointain, ni une Anthropologie seulement faite par des chercheurs qui travaillent avec les autochtones, mais plutôt une façon d'éviter que l'anthropologue soit en danger épistémologique étant qu'un homme occidental et moderne. Cela est une manière de montrer à l'anthropologue la possibilité de mondes multiples et non simplement l'existence de plusieurs visions de mondes.

L'accent sur la différence, comme a bien souligné Sylvie Poirier en apprenant sur les auteurs de ce domaine de recherche, est une tentative d'obliger les chercheurs à penser de façon relationnelle. En considérant le concept de «diversité», on remarque qu'il est encore l'expression de la dichotomie entre l'universel et le particulier. Pour qu'on puisse avoir de la diversité, il faut l'existence d'un universel qui réponde à cette diversité, c'est-àdire il est nécessaire que les diversités fassent partie d'un même monde, la question qui se pose est donc qui a le droit de dire comment est LE MONDE. La différence amène à la multiplicité qui nous oblige, alors à penser aux relations. Ce n'est pas différentes visions de monde, mais plutôt des mondes multiples.

Nous pouvons penser que ce n'est qu'une question de jeu conceptuel qui, à la fin du processus, ne change en rien la pratique anthropologique, mais cela seulement dans le cas où on n'arrive pas à comprendre la proposition épistémologique de replacement du chercheur à laquelle cette approche nous invite. Le propos nous invite à questionner la dimension du pouvoir qui est intrinsèque à la relation chercheur/interlocuteurs; moderne/non moderne. Cette approche est une invitation à un déshabillement du concept déjà prêt, elle est aussi un appel au chercheur à comprendre non seulement les mondes des natifs, mais en même temps son propre monde. 
Bruno Latour (1994) montre que la condition moderne génère le processus de dichotomie entre nature et culture, qui produit à la fois les hybrides en prolifération (même si la société moderne essaie de nier cela), ce qui crée une impasse dans ce qu'on appelle «l'environnement». La Constitution moderne selon l'auteur «invente une séparation entre le pouvoir scientifique chargé de représenter les choses et le pouvoir politique, c'est-à-dire celui chargé de représenter les sujets, ainsi que la séparation entre nature et culture» (Latour: 35). Suite à cette relation dichotomique entre nature et culture qui est présente dans cette constitution moderne, on comprend le processus de domestication de la nature et en même temps, le processus de sa destruction. Cependant, dans une logique aussi non-dichotomique Bateson remarque que la société moderne finit par se détruire soi-même. Dans ce sens, les catastrophes écologiques ne sont plus que les résultats d'une constitution moderne qui est fragmentée.

L'écologie de la vie proposée par Tim Ingold (2000) fixe ainsi comme propos «non-moderne» la négation de la dichotomie entre nature et culture, l'organisme et la personne doivent être considérés comme un seul et même élément d'analyse, ce qui signifie arrêter d'essayer de reconstruire l'être humain complet à partir de deux éléments distincts (corps et esprit).

Tim Ingold dialogue avec Gregory Bateson et Merleau Ponty dans le domaine de la phénoménologie, en essayant de comprendre ces processus cognitifs par rapport à l'environnement, son concept de skill est donc né, celui-ci est défini par l'auteur non pas comme des techniques du corps, mais comme des facultés de perception et d'action d'un organisme quelconque, situé dans un environnement. Ainsi la propriété des organismes humains sont à la fois biologique et culturelle, parce que les relations sont de l'ordre écologique.

La critique d'Ingold à Bateson et aussi à Csordas se pose dans la non problématisation de la dichotomie Nature-Culture. Bateson rompt avec la dichotomie corps/esprit en démontrant que l'esprit ne se limite ni au cerveau ni au corps, qu'il se produit à travers un processus de perception de l'environnement, dans cette perspective Bateson dissout donc la dichotomie entre raison et émotion, mais ni lui, ni Csordas, arrivent à rompre avec la dichotomie Biologie / Culture. 
Pour Tim Ingold (2000) il ne suffit pas seulement de concevoir la nature comme une construction de la culture, en gardant la séparation du corps et de l'environnement. Le concept d'organisme-personne mine ces différences parce qu'il comprend non seulement l'être humain dans l'environnement, mais aussi, les êtres humains non seulement humain, culturel et biologique, mais ceux qui sont résultat de la fusion de l'organisme, de la personne et de l'environnement.

Cette séparation entre nature et culture proposée par Tim Ingold est considérée comme une tension nécessaire par Csordas, la question n'est pas de dichotomiser ou de rompre définitivement avec ces frontières, mais plutôt de travailler dans la tension de ces frontières.

Dans ce sens, nous entrons dans une impasse qui est constitutive de cette «nouvelle anthropologie», celle de la question politique. Dans une tendance de toujours distinguer le local du mondial, la nature de la culture, le mien et le tien (des séparations qui sont dignes de la constitution de la modernité), on met les «problèmes du monde» toujours sur le dos des autres, comme suggère Bruno Latour (1994). Ainsi les auteurs posent la question : Quand sommes-nous «L'autre»? Quand le global est-il dans le local? Quand la nature est-elle dans la culture? Quand la culture est-elle dans la nature?

La nécessité de travailler la tension proposée par Csordas réifie la question qu'il ne suffit pas de refuser la dichotomie, mais de la remettre tout le temps en question. C'est là où réside l'importance de l'anthropologue contemporain, c'est à travers l'ethnographie, dans le plan empirique qu'on travaille dans cette tension épistémologique que suggère Csordas.

Inspirée par Bruno Latour, l'hybridisme, l'organisme à la fois culturel et naturel qui est produit au milieu de la négation de sa propre existence - est le focus du regard que j'essaie de délimiter dans ce travail. Si on pense la Constitution Moderne et toute sa relation d'objectification du monde, du contrôle des corps et de l'État de droit, on pourrait apercevoir que toute cette normalisation produit son propre paradoxe. Les hybrides sont ceux qui n'arrivent pas à être catégorisés par la société moderne, ceux qui échappent ou même résistent à son imposition.

J'invite les lecteurs à une promenade dans une rue de Porto Alegre, ville située au sud du Brésil où j'ai développé mon terrain de maîtrise pour qu'on puisse penser cette 
approche dans le milieu urbain. La rue s'appelle Rua da Praia (Rue de la Plage). C'est une des plus importantes rues où toute la construction de cette ville a commencé. Son importance est à la fois historique, économique et symbolique. Depuis l'arrivée des premiers couples des Açores en 1752, cette rue a été le chemin le plus important par où la ville s'est développée.

Dans le passé, les images de la Rua da Praia apparaissent dans les paroles des chroniqueurs de Porto Alegre comme un lieu de glamour, une rue majestueuse qui abritait des personnages célèbres, venus des milieux les plus favorisées économiquement, qui se promenaient élégamment par les trottoirs, en faisant du footing.

Toute la région centrale de Porto Alegre, mais plus particulièrement la Rua da Praia, était l'espace des intellectuels et de la haute société. Une place pour les belles filles qui se promenaient en chapeaux et flirtaient avec des citoyens honorables. Ce scénario d'ailleurs a radicalement changé dans les temps d'aujourd'hui. Le phénomène qu'on appelle «dégradation du Centre-ville » a été sujet de discussion de plusieurs institutions et agences gouvernementales. Les acteurs sociaux qui sont là ont changé et maintenant, au lieu d'un groupe urbain marqué par son «pouvoir de capital économique», selon Pierre Bourdieu (2007), l'espace est occupé par la «classe populaire», c'est-à-dire les acteurs sociaux dépourvus de ce capital culturel et économique.

La Rua da Praia n'est plus constituée de cafés, de glamoureuses pâtisseries fréquentés par la haute société, ni même de boutiques élégantes qui présentaient autrefois dans leurs vitrines les dernières tendances de la mode. Ses trottoirs ont été pris par des tapis en plastique qui recouvrent les ondulations en noir et blanc tracées sur le sol, et sur lesquels on trouve des articles comme DVD, vêtements, chaussures, poupées, parapluies et d'autres accessoires fabriqués en Chine. La rue est devenue un grand centre commercial de la classe populaire, une fois que les produits sont disponibles à bas prix par les vendeurs de rue organisés illégalement, ce qu'on appelle les camêlos.

Le lieu qui continue marqué par des turbulences culturelles et économiques, s'est ainsi transformé. Au lieu de longues robes, des talons hauts, des chapeaux, des chaussures cirées et des parapluies qui protégeaient les dames de l'action des rayons solaires dans les années 50, nous trouvons des pantoufles, des shorts, des chemises qui sont exposés dans la « vitrine populaire »-par terre. Toutefois, elle a encore révélé « le centre » ville. 
La Rua da Praia d'aujourd'hui est marquée par la confluence des groupes hétérogènes. Les passants sont de tous les milieux sociaux, certains plus que d'autres se conforment en tant qu'habitués de cet espace public, où ils socialisent et construisent leurs identités en tant que personnages faisant partie du «centre». Dans le centre de Porto Alegre, il y a une prédominance d'activités commerciales formelles et informelles, où les sujets issus des «couches populaires » ou de classes moyennes-populaires, c'est-à-dire ceux qui font partie d'une majorité qui n'a pas de pouvoir d'achat, exercent les métiers de vendeurs de journaux et de magazines, fleuristes, retraités, gestionnaires de magasins, réceptionnistes, travailleurs du secteur tertiaire, cireurs de chaussures, vendeurs informels : tels que les artisans, les vendeurs ambulants et les prostituées. Ce sont les personnages qui constituent la majorité des passants dans cette rue, soit comme travailleurs, soit comme habitués de l'espace.

La situation de cette rue en 2008 (année de mon terrain) n'était pas celle que les gouverneurs et ses «citoyens » voulaient de ce lieu de grande importance symbolique et historique de la ville. Les rues du centre de Porto Alegre à la fin du $\mathrm{XX}^{\mathrm{e}}$ siècle et du début du XIX $\mathrm{X}^{\mathrm{e}}$ ont été conçues dans le but de démontrer l'existence de la modernité, qui visait, à son tour, une classe sociale spécifique, ce que nous pourrions envisager aujourd'hui comme une «classe moyenne/aisée», mais souligne Pesavento (1996: 38): «la rue reflète la transformation de l'espace urbain et la réorganisation de la vie». Ainsi, les rues du centre de Porto Alegre ont été peu à peu réappropriées et ré signifiées par les habitants de cette ville à travers leurs pratiques quotidiennes en réorganisant la logique qui a été initialement conçue par le gouvernement.

Selon Ruben Oliven (1980), dans les études de sociétés complexes, le milieu urbain présente une homogénéisation apparente des classes sociales dans les centres urbains en raison de l'intensification du capitaliste industriel. L'auteur souligne, cependant, les dangers de cette analyse qui ne tient pas compte du fait que ce processus d'accumulation du capital distingue les habitants des sociétés brésiliennes de façon inégale et asymétrique, mais que les classes inférieures, elles aussi, peuvent résister à la diffusion de ces «orientations culturelles standardisées». Dans ce sens, Oliven (1980) affirme que les différents groupes sociaux ont différentes pratiques en ce qui concerne «les aspects qui 
ont des conséquences et des significations diverses d'après la position sociale comme, par exemple, les questions d'ordre politique » (1980: 35)

Les centres urbains, selon Antônio Augusto Arantes dans son analyse de la ville de São Paulo, ont une grande tendance à l' «accroissement de logements et aussi un plus grand nombre de personnes qui dort dans les rues du centre-ville » (2000: 144). Pour l'auteur, ce cadre est dû à l'appauvrissement élevé des classes inférieures, ce qui déclenche des «paysages où la pauvreté vernaculaire et la différence culturelle - dans ses plusieurs faces - définissent et situent socialement les façades de cristal globalisé, qui à leur tour les reflètent en politisant l'espace urbain (2000: 145) ».

Ce déplacement de la classe populaire vers les centres urbains met en évidence la tension exercée sur l'espace urbain, y compris celle exercée sur la Rua da Praia. D'une part, un débat patrimonial qui vise à sauvegarder les monuments, les places, les bâtiments historiques à travers un processus de «rééducation patrimoniale pour l'utilisation de l'espace public», comme le projet du gouvernement fédéral appelé Monumenta ${ }^{2}$ a exigé. D'autre part, la vie quotidienne de ces habitués, qui connaissent cet espace urbain et s'approprient de ces «points d'amarrage » de la mémoire de la ville (Arantes, 2000), à travers leurs pratiques quotidiennes et leur sociabilité, affronte la logique de préservation du patrimoine. En dialogue encore avec Antonio Arantes, on remarque que ce conflit est immergé dans un ensemble complexe de significations et représentations dans l'ordre du vécu et aussi de l'officiel, on ne peut pas considérer ce phénomène d'une manière unilatérale et simplifiée qui présuppose une simple accommodation de la classe populaire à ce système de règles et de conduites qui sont établis par des organismes officiels pour l'utilisation de l'espace public », parce que les «représentations du centre-ville faites par ceux qui habitent ses places et ses rues ne sont pas différentes de celles des monuments du paysage officiel, bien au contraire, tous les deux articulent leurs expériences sociales à un espace, en lui conférant un contexte et des significations populaires » (2000: 122).

\footnotetext{
${ }^{2}$ Le programme Monumenta est un programme du gouvernement fédéral brésilien. C'est une initiative du Ministère de la Culture de ce pays qui réunit 27 villes du territoire fédéral. Le programme vise à «revitaliser» les centres urbains brésiliens à partir des initiatives de conservation du patrimoine matériel et des politiques de rééducation patrimoniale qui a pour objectif rééduquer les habitués actuels de ces centres pour qu'ils vivent en accord avec la logique de préservation patrimonial.
} 
Le conflit qui a été ici exposé signale une bataille urbaine, une dispute d'un territoire, ou plutôt une dispute de l'État pour que les groupes urbains soient encadrés dans une logique du tourisme, dans une logique de préservation, et que la rue soit prévue comme objet qui est décharné de la façon de vivre ce lieu. Est-ce que les paysages d'une ville sont en dispute? Est-ce que les concepts distincts de villes sont en jeu? C'est dans ces différents mondes où la modernité à travers son représentant suprême, l'État, essaie de nier la différence en transformant la multiplicité d'image en une seule image, celle de la modernité, du progrès et de son patrimoine matériel.

Ça ne suffit pas de parler d'une logique de l'État, on doit aussi se demander de quelle manière cette logique se déroule-t-elle? Va-t-elle à la rencontre de quels intérêts? La Constitution de l'État Moderne sert aux intérêts de quels citoyens? Qui sont ces citoyens? Que veut dire ce mot, trop diffusé, dans le monde moderne? Au premier regard, les citoyens seraient tous ceux qui font partie d'un État Nation. Les citoyens ont leurs droits constitutionnels que l'état du bien-être social devrait garantir. Dans ce cas, plusieurs concepts doivent être revus. Que signifie un état de bien-être? Est-ce que cela a le même sens pour tous les « citoyens »? La définition de «bien-être» est définie par la Constitution Moderne (Latour, 1994), donc qui dichotomise les choses et essaie de toujours «nettoyer », purifier, classifier et surtout ne pas mélanger les éléments qui font partie de la vie. Dans ce «monde » moderne on rencontre d'un côté, la nature qui doit être dominée et, de l'autre, la culture, surtout l'occidentale, qui domine la nature (et bien sûr, toutes les autres cultures). Dans cette logique, il y a les pays développés, riches qui doivent «aider» les pauvres, «les primitifs»; les citoyens-consommateurs qui se heurtent à cette logique moderne, ceux qui veulent une rue bien aseptisée de la pauvreté et du paradoxe de leur propre modernité, et les habitués locaux qui essaient toujours de suivre leurs vies, de continuer à exercer leurs pratiques quotidiennes, ceux qui résistent à être eux-mêmes.

Le processus de «revitalisation» des centres urbains brésiliens peut être analysé comme une tentative d'éliminer, au moins de l'espace public central, historique et aussi touristique, l'image du désordre, l'image de la pauvreté, l'image qui montre qu'il y a encore des citoyens qui ne sont pas autant citoyens que les autres. L'interdiction du commerce informel et la possibilité de l'existence des clôtures des places mine la façon de 
se sociabiliser dans cet espace public, sape la forme dont les habitués locaux se rapportent entre eux et avec l'environnement.

Le projet d'urbanisation et de « revitalisation » des espaces publics part du principe qu'il n'y a plus de vie dans ce lieu. Le mot « revitaliser» qui est utilisé par l'État pour désigner les œuvres qui visent transformer les lieux, les places ou même les bâtiments en patrimoines historiques de plusieurs centres urbains brésiliens, y compris à Porto Alegre, indique l'intention de donner, de nouveau, la vie à quelque chose qui n'en a plus, à quelque chose qui est déjà morte. Quelle vie la ville doit-elle avoir? Quelle vie est-elle digne d'être considérée comme vivante ? Est-ce qu'il n'y avait pas de vie qui pulsait intensément dans ce lieu? Considérer qu'il n'y a plus de vie à Rua da Praia, ou à n'importe quel lieu qui passe par ce processus de revitalisation, c'est concevoir une idée de vie idéale qui doit être vécue dans un lieu idéal, où l'idéal de vie et de ville est imposé par le modèle de la modernité, celui qui sert à tout citoyen à condition qu'il soit consommateur.

Le processus de «revitalisation» n'est pas une grande nouveauté dans les villes contemporaines. Au Brésil, par exemple, il y a eu pendant les années 60 un grand processus appelé à l'époque processus «d'hygiénisation», c'est-à-dire de «nettoyage» culturel et social des villes. Un nettoyage dans le sens de ôter la pauvreté des yeux de ceux qui étaient gênés. A cette époque plusieurs complexes d'habitation populaire ont été créé le plus loin possible du cœur des villes. L'intention d'enlever tous ceux qui n'avaient pas la même façon d'être et de se comporter dans le milieu urbain est une façon de légitimer ceux qui sont considérés comme les citoyens, les vrais citoyens, c'est-à-dire les gens qui sont déjà bien adaptés et qui vivent d'après la constitution moderne.

Il est important de remarquer que la classe populaire n'est pas limitée uniquement par sa situation socio-économique. Faire partie de la classe populaire ne veut pas seulement dire avoir un bas pouvoir d'achat, cela dénonce aussi des styles de vies différents de ceux prévus par la modernité. La classe populaire est l'espace du bricolage, de la réinvention du quotidien, d'une lutte constante pour la survie.

Il est important de penser au sens du mot «populaire». Quand Cavalcanti (2010) envisage le carnaval comme une fête de la culture populaire, un événement de caractéristiques populaires, il fait aussi la problématisation du mot «populaire ». Si on pense la dimension populaire ajoutée au caractère urbain de la vie contemporaine, on 
pourra comprendre que les couches populaires ne peuvent pas être réduites à une condition économique. L'auteur explique que le populaire ne doit pas être pensé seulement en contraposition à la culture d'élite. Dans les sociétés complexes, les acteurs sociaux jouent avec les stéréotypes et déclenchent leurs identités selon leurs nécessités, la dimension populaire est ici aussi une dimension politique qui dévoile les conflits sociaux.

Pierre Sansot (1986), en parlant aussi de la culture populaire, affirme que «la culture populaire a été le lieu d'élection du sensible. Y vivaient des hommes qui vivaient, pour leur travail, ils ne décollaient jamais du réel et pour lesquels le plaisir naissait d'une exaltation ou d'une quiétude de sens et non de soupirs métaphysiques ou de sentiments distingués » (1986: 20).

D'après Gilberto Velho (2004), les grandes villes ont passé par un violent processus de modernisation qui affecte sérieusement le système de valeurs et les rapports sociaux. L'expansion de l'économie de marché, l'industrialisation, la migration et l'enrichissement de la culture de masse ont contribué pour la croissance de l'individualisme. Dans ce processus, les idéologies individualistes ont gagné de l'espace, le champ de possibilités socioculturelles, les alternatives et les choix de style de vie se sont diversifiés. Selon l'auteur, le secteur populaire et plusieurs minorités ont commencé à avoir plus de reconnaissance et de présence dans la société, même s'ils ont payé cher pour cela. Ainsi, la diffusion des valeurs individualistes a signifié un affaiblissement des formes traditionnelles de domination, associé à une vision de monde hiérarchique. Simmel (1979) a déjà bien discuté la notion d'individualisme au sein de la société moderne comme l'espace de la compétition et aussi du conflit, mais aussi l'espace de sociabilité. Le conflit, comme nous enseigne l'auteur, est déjà une façon de se socialiser. Le conflit peut être analysé comme une manière de mettre en contact, en dialogue, des mondes distincts, ainsi il n'est pas toujours négatif, au moment où il peut produire un dialogue entre cultures, une sociabilité qui tient compte de la différence.

Malgré ce processus d'individualisation et d'intensification de la modernité et du «sujet moderne», la ville continue l'espace où habitent les différences et où on abrite aussi les sujets qui ne s'identifient pas avec la condition moderne, ceux qui ne sont pas représentés par l'idée d'individu. La ville est le lieu de la multiplicité de groupes culturels qui sont en dialogue. Ces diverses cultures en contact déclenchent le conflit qui est 
implicite à cette sociabilité. La dimension urbaine, les différents modes de vie qu'abritent la ville et la forme dont les distincts groupes sociaux se disputent pour la manutention et la préservation de sa façon «d'être au monde» (en utilisant leurs stratégies et leurs astuces quand ils sont en dialogue avec l'État ou n'importe quelle autre institution qui essaie de nier la façon d'être) ont toujours été ma préoccupation anthropologique majeure.

Dans ce sens, au doctorat je me pose la même question d'une autre façon. En analysant les carnavals du Québec/Canada et aussi le carnaval de Rio de Janeiro/Brésil on est capable d'apercevoir un grand processus de «purification» (en utilisant un euphémisme) de ces fêtes populaires. Une tentative de conformation de ces fêtes à l'expectative des touristes consommateurs qui la profite. La commercialisation de la culture populaire a déjà été objet de grande discussion, mais il faut aussi regarder de plus près ces relations et voir de quelles manières tous les acteurs (le gouvernement, les touristes et, principalement, le peuple qui vit par et pour ces fêtes) répondent face au jeu d'innovation et de tradition qui fait partie de la dynamique de perpétuation de ces fêtes.

Dans le carnaval de Québec comme dans celui de Rio de Janeiro, les États savent qu'une image du pays est projetée à l'étranger. Dans ce sens, le gouvernement essaie de bien formuler cette image au goût de son public. L'image d'une fête carnavalesque n'est pas une image quelconque, c'est l'image du plaisir et du ludique, c'est une image qui a un appel touristique et qui invite les gens à s'amuser pendant ces événements et par conséquence à connaître les pays où ces carnavals se déroulent. L'alcool, toujours lié au dionysiaque, a un rôle important dans ces rituels urbains parce que c'est aussi à travers lui qu'on peut apercevoir le jeu d'interaction entre le gouvernement et «son peuple».

Ici, à Québec, dans le carnaval 2012, je me souviens d'une publicité qui montrait les cannes du bonhomme du carnaval sans le couvercle (la tête du bonhomme) avec cette phrase: «Dans ce carnaval ne perdez pas la tête ». Même s'il est interdit, les personnes remplissaient leurs cannes avec de l'alcool pour s'amuser au carnaval.

Les rituels jouent avec l'imaginaire d'un Québec froid et discipliné. Ce mouvement des joueurs avec les règles sociales n'est pas une exclusivité du peuple, mais aussi du gouvernement. Le slogan du carnaval 2012 ( «le carnaval montre ses couleurs ») est une tentative de démystifier l'idée de l'hiver dépressif et montre de quelle manière cette saison peut être amusante et intéressante. 
Dans le cas du carnaval brésilien, les actions gouvernementales jouent aussi avec le préétabli par l'État. Le carnaval c'est le moment où presque tout est permis, où les gens peuvent sortir en criant par les rues et où personne n'appelle la police pour dire qu'elles dérangent l'ordre. La musique résonne au loin jusqu'à très tard dans la nuit avec les éclats de rires des ivrognes. C'est le moment où l'État brésilien distribue plus de préservatifs que d'habitude au peuple parce que neuf mois après ce rituel le taux de natalité est le plus haut.

Avec ces données, les États comprennent que les carnavals vont déclencher cette dynamique de contravention et, en étant au courant de cela, jouent aussi avec l'événement et essaient de diminuer les mauvaises conséquences avec la création de publicités de conscientisation avant et pendant les rituels, en augmentant aussi le nombre de policiers dans les fêtes comme une forme de coercition, cependant les États flexibilisent simultanément les règles d'appréhension soit à cause de l'alcool, soit à cause du bruit.

Ainsi on voit le concept de «poétique sociale » de Herzfeld (1997), comme une forme d'agence qui crée des stratégies d'action et de réaction des groupes sociaux face aux actions de l'État. L'État-nation, selon Herzfeld, a pour fonction « dépoétiser » la vie sociale. Herzfeld avertit que la «poétique sociale» est également en vigueur dans les actions de l'État, mais l'État-nation cherche à effacer les traces de sa propre créativité, qui est inhérente à son existence, créant ainsi des essentialismes. Dans ce sens, l'auteur souligne que la démocratie n'est pas synonyme de plus de tolérance, dans la mesure où, au nom de l'égalité, elle efface les différences sociales et culturelles.

Toutes ces réflexions ont été faites à partir de ma recherche de maîtrise, et celle du doctorat est une tentative de repenser le contexte urbain à la lumière d'une Anthropologique qui dévoile ce paradoxe de la société contemporaine : essayer d'être «propre» et «aseptique», sans mélanger la culture et la nature, sans se laisser contaminer par les contraintes non moderne tout en produisant ses propres hybrides, comme bien souligne Bruno Latour. Dans ce sens, garder la culture populaire est aussi une réponse à cette tentative de purification de la culture, de nettoyage des hybrides.

La fête du carnaval de Québec a changé. Le processus de purification a été un succès. Aujourd'hui quand je parle à un natif à propos du carnaval de Québec celui-ci me répond avec un sourire ce qui dénonce sa dépréciation face à cette fête. Le carnaval de 
Québec a été «purifié », mais comme souligne Sabev (2003) la façon de faire la fête carnavalesque est encore vive chez plusieurs acteurs sociaux.

Le carnaval de Québec est devenu un carnaval familial, comme nous le montre Sabev (2003) et il a exclu de cette façon les jeunes et les adolescents de son espace officiel. La fête est produite pour les enfants et leurs parents. La question que l'auteur se pose c'est que les jeunes qui ne plus enfants et ne sont pas encore adultes sont obligés à faire un autre carnaval, ce qu'ils appellent le «décarnaval». Celui-ci est né de ce conflit entre une construction identitaire d'un carnaval légitime et familier - un carnaval officiel - et un carnaval qui est capable de faire plaisir aux jeunes, un carnaval pas très commercial et pas contrôlé par l'État, sans les barrages de protections policières.

La naissance du décarnaval est aussi une manifestation contre le pouvoir officiel, c'est une façon de montrer que l'identité de ces jeunes ne se forme pas à partir des valeurs et des styles de vie qui veulent être valorisés dans le carnaval officiel. C'est par ailleurs une manière de se battre contre le système. L'État prend ainsi conscience de ces manifestations et est obligé de devenir plus flexible, voire, de fermer les yeux. Il est même à la recherche d'alternatives pour englober ces jeunes.

À Rio de Janeiro le processus de purification a déjà commencé, les batailles gouvernementales pour une image d'un carnaval propre et sûr ont déjà été livrées. Au cœur de cette lutte quotidienne «d'exister» à sa propre façon, les personnages entrent dans le jeu politique et résistent aux impositions.

Suivant les traces de Michel De Certau (2008) nous voyons l'existence d'une dynamique du social qui est articulée par les acteurs sociaux dans leurs vies quotidiennes où la ville est à la fois la scène et l'objet de ces tensions. Ainsi, ces acteurs sociaux y compris la classe populaire, cherchent des stratégies et créent des astuces qui sont négociées dans leurs espaces urbains de la vie quotidienne comme des formes de résistance à cette logique dominante et officielle.

Nancy Munn (2006) en dialogue avec De Certau (2008) nous signale cette dynamique du vécu qui flexibilise les frontières et les prescriptions. En analysant ses données de recherche, elle a quelques préoccupations théoriques du domaine des sciences humaines et sociales qui évoquent les sujets tels que l'espace, le temps et les réactions corporelles en relation avec les espaces et leurs pouvoirs, les discours de position et la 
capacité de se mobiliser, d'être mobile. Pour l'auteur, les frontières sont, dans un sens pratique, comme le mouvement du corps. Les gens en action produisent des frontières, des expériences de frontières, ou encore, ils sont, eux-mêmes, ces frontières. Celles-ci sont mobiles au fur et à mesure que les corps bougent. Nancy Munn encourage cette relation espace-temps en la dynamisant, puis elle montre que ce mouvement dans l'espace géographique qui a lieu dans les rituels est aussi une dimension temporelle, car pour un certain temps les gens sont privés de sortir de certaines régions. Dans ce sens, les «espaces exclus» sont exclus à la fois dans l'espace et dans le temps.

Ce mouvement dans l'espace a un temps, pas nécessairement fixe dans le temps du rituel, mais un temps d'interdiction de certains espaces et aussi un temps pour y demeurer, de sorte que les frontières sont fluides spatiotemporelles.

Ainsi, nous pouvons discuter la question du paysage des exclus. Si on reprend la pensée à propos de la Rua da Praia on peut la voir comme un paysage de litige, et de cette manière constater que dans la mesure où elle se définit aujourd'hui comme un paysage de la classe populaire qui la conforme et y habite, dans les temps d'ailleurs, lorsqu'on faisait du footing, quand cette même rue était l'espace des jeunes femmes de la haute société, cette rue - la Rua da Praia - excluait ceux qui, aujourd'hui, font pulser ce paysage, la classe ouvrière et populaire. Cette interdiction constitue une frontière symbolique que les défavorisés ne pouvaient pas surmonter.

Quand je demandais à mes interlocuteurs de la recherche de maîtrise, qui ont vécu cette rue dans les années 1950 (à l'époque du glamour et de la haute société) quel était le sens de la Rua da Praia pour eux, la réponse était immédiate: c'est de l'amitié, des loisirs, c'est « où nous sommes nous-mêmes », mais si je cherche à reconstruire cette expérience dans cette même rue avec ces mêmes acteurs sociaux actuellement, je n'y arriverais pas parce que le paysage a été transformé, les acteurs sociaux ont changé et par conséquent toutes les pratiques quotidiennes qui en découlent. Les personnages d'ailleurs n'y vivent plus parce que leurs expériences ont changé, la rue a changé, les acteurs ont changé.

J'ai essayé de montrer dans cet article que plusieurs conflits dans le milieu urbain se déroulent du fait que les expériences diverses produisent des mondes différents, alors quand on parle d'une dispute sur la Rua da Praia, par exemple, on doit se rendre compte qu'on parle de plusieurs Ruas da Praia: la rue des habitués des années 50, la rue des 
habitués d'aujourd'hui, la rue que le gouvernement a planifié, la rue que les touristes espèrent voir, la rue que pour quelques-uns est l'espace de loisir pendant que pour d'autres c'est un espace à éviter. De la même façon quand on met en évidence un processus de purification des fêtes populaires comme les carnavals on doit se rendre compte que ce sont plusieurs carnavals qui sont en dispute, et que la société moderne essaie toujours de montrer la vérité. Le vrai carnaval bien comme la vraie Rua da Praia. Un seul monde, un seul lieu et une seule façon de faire la fête. Toutes les variations sont autorisées pourvu qu'elles ne méprisent pas ce qui est préétabli par la condition moderne.

La tentative de purification et de délimitation d'une seule Rua da Praia, comme aussi une seule façon de faire le carnaval, est une tentative moderne de nier les différences et d'établir la vraie manière de regarder et vivre «le monde». Cette problématique a toujours été le cœur de mes questionnements, mais à travers un dialogue fructueux avec ceux que j'avais pensé les «acteurs des autochtones »j'ai été apte à regarder les mêmes sujets en utilisant leurs perspectives épistémologiques. Et, maintenant je suis capable de les voir comme acteurs qui sont au-delà de ces boîtes, qui nous font repenser notre propre statut de chercheur et ainsi nous obligent à revisiter notre propre façon de faire l'anthropologie en regardant toujours l'Autre sans oublier de nous observer.

\section{Références}

ARANTES, Antonio Augusto Neto. Paisagens Paulistanas: transformações do espaço público. Campinas, SP: Editora da Unicamp, 2000.

BATESON, Gregory. Form, Substance, and Difference. In: BATESON, Gregory. Steps to an ecology of mind. Chicago/London, The University of Chicago Press, p. 454-471. 2000.

CAVALCANTI, Maria Laura Viveiros de Castro. Carnaval : o rito e o tempo. ( Carnaval : le rite et le temps) Rio de Janeiro : Civilização Brasileira, 1999.

CRAPANZANO, Vincent. El dilema de Hermes: la máscara de la subversión en las descripciones etnográficas. In: CLIFFORD, James y MARCUS, George E. (Eds.). Retoricas de la antropologia. Madrid, Ediciones Júcar. p. 91-122. 1991.

CRAPANZANO, Vincent. "Imaginative Horizons" $e$ "The Between". In: CRAPAZANO, Vincent. Imaginative Horizons. An Essay in Literary-philosophical Anthropology. Chicago/London. The University of Chicago Press, p. 13-65. 2004. 
CSORDAS, Thomas. A Corporeidade como um Paradigma para a Antropologia. In: CSORDAS, Thomas. Corpo/Significado/Cura. Porto Alegre, Editora da UFRGS, 2008, p. 101-146 (no prelo). De Certeau, Michel. A Invenção do Cotidiano. Petrópolis, RJ : Editora Vozes, 2008 .

DESCOLA, Philippe. Le grand partage. In: DESCOLA, Philippe. Par-delà nature et culture. Paris, Gallimard, p. 91-131. 2005.

HERZFELD, Michael. Cultural Intimacy: Social Poetics in the Nation- State. New York/ London: Routledge, 1997.

INGOLD, Tim. The perception of the environment. Essays in livelihood, dwelling and skill. London/New Yor, Routledge, p. 9-60. 2000.

JULLIEN, François. De l'universel, de l'uniforme, du commun et du dialogue entre les cultures. p. 127-159. Paris : Fayard. 2008.

LATOUR, Bruno. Jamais fomos modernos. Rio de Janeiro, Ed. 34, p 149. 1994.

MUNN, Nancy. Excluded Spaces: the Figure in the Australian Aboriginal Landscape. In: SETHA, Low and LAWRENCE-ZÚÑIGA, Denise. The Anthropologu of space and place. Oxford, p. 92-109. 2006.

OLIVEN, Ruben George. O nacional e o estrangeiro na construção da identidade brasileira. In: Bernd, Zilá (org.). Olhares Cruzados. Porto Alegre: Editora UFRGS, 2000.

OLIVEN, Ruben George. Por uma Antropologia em cidades brasileiras. In: Velho, Gilberto. O Desafio da Cidade. Rio de Janeiro: Editora Campus, 1979.

ORTNER, Sherry. Uma atualização da Teoria da Prática" e "Poder e Projetos: Reflexões sobre a Agência. In: GROSSI, Miriam; ECKERT, Cornelia e FRY, Peter. Conferências e diálogos: saberes e práticas antropológicas. Blumenau, Nova Letra/ABA, p. 19-80. 2007.

ORTNER, Sherry. Subjectivité et critique culturelle. In Yacine Tassadit (dir.), Anthropologie et subjectivité, pp. 21-44. Paris : Maison des sciences de l'homme. 2005.

SAHLINS, Marshall. $O$ "pessimismo sentimental" e a experiência etnográfica: por que a cultura não é um "objeto" em via de extinção (Parte I). Rio de Janeiro, Mana, v. 3, n. 1, p. 41-73. 1998.

SANSOT, Pierre. Les formes sensibles de la vie sociale. Presses Universitaires de France, 1986.

SIMMEL, Georg. A metrópole e a vida mental. In: O Fenômeno Urbano. Otávio Guilherme Velho (org.). Rio de Janeiro: Ed. Guanabara,1985.

SIMMEL, Georg. A Filosofia da paisagem. Tradução de Simone Carneiro Maldonado. In: Revista e Política e trabalho, número 12, 1996.

STRATHERN, Marilyn. Out of context: the persuasive fictions in Anthropology._Current Anthropology, v. 28, n. 3, p. 251-281. 
STRATHERN, Marilyn. Estratégias antropológicas. In: STRAHERN, Marilyn. O Gênero da Dádiva. Problemas com as mulheres e problemas com a sociedade na Melanésia. Campinas, Ed. Unicamp, p. 27-51. 2006.

TODOROV, Tzvetan. A conquista da América. A questão do outro. São Paulo, Martins Fontes. Parte I, p. 3-48 e 241-250. 1991.

VIVEIROS DE CASTRO, Eduardo. O nativo relativo. Rio de Janeiro, Mana, v. 8, n. 1, p. 113148. 2002.

Les pronoms cosmologiques et le perspectivisme amérindien. In Éric Alliez (dir.), Gilles Deleuze. Une vie philosophique. Paris : Le Plessis-Robinson. p. 429-462. ISBN : 2843240220 (576p.). 1998.

Métaphysiques cannibales. p. 77-92; 159-169. Paris: PUF. 2009.

Recebido em: 05/09/2013

Aprovado em: 18/10/2013 